\title{
Surface microfluidics fabricated by photopatternable superhydrophobic nanocomposite
}

\author{
Lingfei Hong $\cdot$ Tingrui Pan
}

Received: 10 September 2010/ Accepted: 27 October 2010/Published online: 16 November 2010

(C) The Author(s) 2010. This article is published with open access at Springerlink.com

\begin{abstract}
Surface microfluidics can be of potential use in a variety of emerging applications, including biological and chemical analysis, cellular detection and manipulation, high-throughput pharmaceutical screening, and etc. In comparison with the conventional closed-channel microfluidic system, surface microfluidics shows the distinct advantages of simple construction, direct surface access, no cavitation or interphase obstruction, clear optical path, easy fluidic packaging, and device reusability. In this article, we first present surface microfluidic networks microfabricated by a single-step lithographic process using a novel superhydrophobic photosensitive nanocomposite formula. The photopatternable superhydrophobic nanocomposite (PSN) incorporates PTFE nanoparticles into a SU-8 matrix, in which superhydrophobicity (contact angle of above $160^{\circ}$ ) is primarily contributed by the extremely low chemical energy and nano-topology of PTFE nanoparticles, while the SU-8 polymer matrix offers photopatternability (lithographic resolution of $10 \mu \mathrm{m}$ ) and substrate adhesion. Moreover, an additive intermediate layer with hydrophilic sidewall considerably reduces flow resistance while improving the substrate adhesion, as a crucial improvement from the previous surface flow configuration. Furthermore, self-propelled microfluidic networks driven by surface tension-induced pressure gradient have been fabricated and characterized to demonstrate the applicability of the novel nanocomposite fabrication approach.
\end{abstract}

\footnotetext{
L. Hong · T. Pan $(\bowtie)$

Department of Biomedical Engineering, University

of California, Davis, USA

e-mail: tingrui@ucdavis.edu

L. Hong

School of Instrumentation Science and Opto-electronics

Engineering, Beihang University, Beijing, China
}

Keywords Surface microfluidics - Superhydrophobicity · Photolithography · Microfabrication · Nanocomposite

\section{Introduction}

Microfluidic systems have been successfully applied to biological and chemical analysis, cellular detection and manipulation, high-throughput pharmaceutical screening, and high-precision optics over the past decades (Gravesen et al. 1993; Beebe et al. 2002; Whitesides 2006), due to significantly reduced chemical consumptions, considerably accelerated reaction rates, as well as highly precise fluidic operations (Bertsch et al. 2001; Wolfe et al. 2004; Piorek et al. 2007; Watanabe 2009b). As an emerging alternative to the conventional microfluidics, surface microfluidic networks, defined by surface micropatterns with sufficient wettability contrast (i.e., hydrophobic patterns on hydrophilic substrate, or vice versa) have intrinsically overcome several technical challenges encountered in the closed-channel counterpart (Piorek et al. 2007; West et al. 2007; Watanabe 2009b). For instance, the exposed gas-liquid interface completely eliminates bubble trapping and cavitation obstruction (Skelley and Voldman 2008; Watanabe 2009a). Furthermore, introducing the large area-to-volume ratio to the open surface platform enables rapid establishment of equilibrium with the surrounding environment, which can be explored to detect specific environmental chemical/physical stimuli (Piorek et al. 2007; Watanabe 2009b). In addition, the dedicated device packaging (e.g., alignment and bonding) and the special macro-to-micro interconnect become unnecessary in many cases, which further simplifies the microfabrication procedure (Piorek et al. 2007).

Surface microfluidics, originally evolved from the surface-directed channels, can be categorized into three 
configurations: the microflow confined between two surfaces of identical hydrophobic micropatterns (Lam et al. 2002; Besson et al. 2006; Lee et al. 2006; West et al. 2007; Watanabe 2009a; 2010), the microflow restricted between one wettability-patterned surface and another uniform hydrophobic substrate (Bouaidat et al. 2005), and the microflow directed on a single planar substrate with ultrahigh wettability contrast (Juncker et al. 2002; Piorek et al. 2007; Hong and Pan 2010a, b), as will be compared in Sect. 3 (Open Surface Fluidic Configurations). The surface wettability-defined microfluidic network was first demonstrated by establishing hydrophobic boundaries on regular glass slides for capillary electrophoresis (Oh 1999). Further investigations on the surface-directed microflow extended to different fabrication techniques, intriguing interfacial phenomena as well as various new applications (Piorek et al. 2007; Swickrath et al. 2009; Yang et al. 2009). Among those, Zhao and his colleagues showed that two immiscible liquids flowed along the wettability boundary instead of following the solid geometric confinement, which was photo-defined by self-assembled hydrophobic monolayer in closed microchannels (Zhao et al. 2001). Furthermore, Watanabe presented that microfluidic components (e.g., channels and reservoirs) can be printed onto a pretreated superhydrophobic glass substrate using an office inkjet printer with hydrophilic inks (Watanabe 2009a, b, 2010). To create wettability contrast, Lipowshy's group deposited a thin layer of $\mathrm{MgF}_{2}$ onto a hydrophobic silicone rubber or a thiolated gold substrate through a shadow mask (Gau et al. 1999). Moreover, hydrophilic patterns can be formed on hydrophobic substrates by direct writing with a microplasma jet or removing hydrophobic features by lift-off technique (Bouaidat et al. 2005; West et al. 2007). In addition, contact printing using PDMS stamps was also implemented to pattern hydrophobic molecules onto hydrophilic substrates (Juncker et al. 2002). Although the aforementioned microfabrication techniques were developed or improved for various utilities of surface microfluidics, none of them completely addressed simple and inexpensive processing, high-definition micropatterning, and universal surface adaptability as a whole, which are of essential importance to build functional surface microfluidic networks on a desired substrate for complex biological/chemical operations.

In this article, we present a single-step photolithography process to devise the surface microfluidic systems using a novel photopatternable superhydrophobic nanocomposite. The novel nanocomposite formula incorporates PTFE nanoparticles into a photosensitive SU-8 matrix, in which superhydrophobicity is primarily contributed by the extremely low chemical energy and nano-topology (i.e., both particle size and spherical shape) of PTFE nanofiller (with contact angle of above $160^{\circ}$ ), while SU-8 offers photopatternability (with the lithographic resolution of $10 \mu \mathrm{m}$ ) and substrate adhesion. Moreover, an additive intermediate layer with hydrophilic sidewall considerably reduces flow resistance while improving the substrate adhesion, as an improvement from the previous surface flow configuration. Importantly, unlike the conventional microflow in spatially enclosed spaces, distinct surface microfluidic manipulations have been observed through adjustment of defined surface geometry and/or control of interfacial profile. Based on the unique open fluidic platform, self-propelled microfluidic network driven by surface tension-induced pressure gradient has been performed and analyzed on the micropatterned superhydrophobic surfaces.

\section{Methods}

\subsection{Photopatternable superhydrophobic nanocomposite}

Photosensitive superhydrophobic nanocomposite (PSN) formula consists of dispersed PTFE nanoparticles of 200-300 nm in diameter (microdispers-200, Polysciences) into SU-8 2000.5 photoresist (Microchem) at a weight ratio of $1: 15$. Before use, ultrasonication $(40 \mathrm{~W}, 20 \mathrm{~min})$ is applied to the mixture, which ensures uniform dispersion of the nanocomposite formula. The PSN combines the hydrophobicity and nanotopology of PTFE filler (surface free energy of $18.6 \mathrm{mN} / \mathrm{m}$ ) (Janczuk and Bialopiotrowicz 1990 ) with the photopatternability and adhesive property of SU-8 matrix, which enables simple fabrication of surface microfluidics on various substrates.

\subsection{Microfabrication of surface microfluidics}

Surface microfluidics utilizes the high-contrast wettability boundary along the superhydrophobic micropatterns on the hydrophilic substrate, also known as the interphase triple lines, to confine the aqueous flow (Gennes et al. 2002). The PSN processed by single-step photolithography can be employed to establish non-wettable micropatterns on the target substrate. Although glass substrate is chosen in this study for its intrinsic hydrophilicity, we have fabricated the PSN micropatterns on various substrates to which SU-8 chemistry can be applied, including polyethylene terephthalate (PET), polydimethylsiloxane (PDMS), and polystyrene (PS). Before coating, the substrate is thoroughly cleaned in a piranha solution $\left(3: 1 \mathrm{H}_{2} \mathrm{SO}_{4}: \mathrm{H}_{2} \mathrm{O}_{2}\right)$ for $30 \mathrm{~min}$, followed by a dehydration bake at $200^{\circ} \mathrm{C}$. As illustrated in Fig. 1a, an additive layer of SU-8 2050 (with the thickness ranging from 15 to $60 \mu \mathrm{m}$ ) can be first deposited as the channel sidewalls to enhance hydrophilicity to aqueous flow once surface-activated. In the following step, the PSN is spin- or spray-coated onto the 
substrate. Spray coating offers greater adaptability and flexibility to substrates with arbitrary geometries, and therefore, is preferred in our procedure (Fig. 1b). Subsequently, the stacked layers of photosensitive SU-8 and nanocomposite formula are patterned together through backside UV exposure of $35-135 \mathrm{~mJ} / \mathrm{cm}^{2}$ (Fig. 1c). After post-exposure bake at $95^{\circ} \mathrm{C}$ and development in a regular SU-8 developer, the unexposed nanocomposite area dissolves, leaving the crosslinked superhydrophobic micropatterns on the surface (Fig. 1d). An oxygen plasma treatment at $30 \mathrm{~W}$ for $30 \mathrm{~s}$ is successively performed to render the sidewall hydrophilicity. As a result, the hydrophilic SU-8 sidewalls could significantly reduce the fluidic resistance in addition to improving coating adhesion to the substrate. In addition, we keep the treated hydrophilic surfaces always wetted (filling with water) to prevent the hydrophobic recovery (Walther et al. 2007). Using the PSN, surface microfluidic networks can be simply constructed on any desired substrate with optical transparency. Moreover, additional water reservoirs can be placed next to
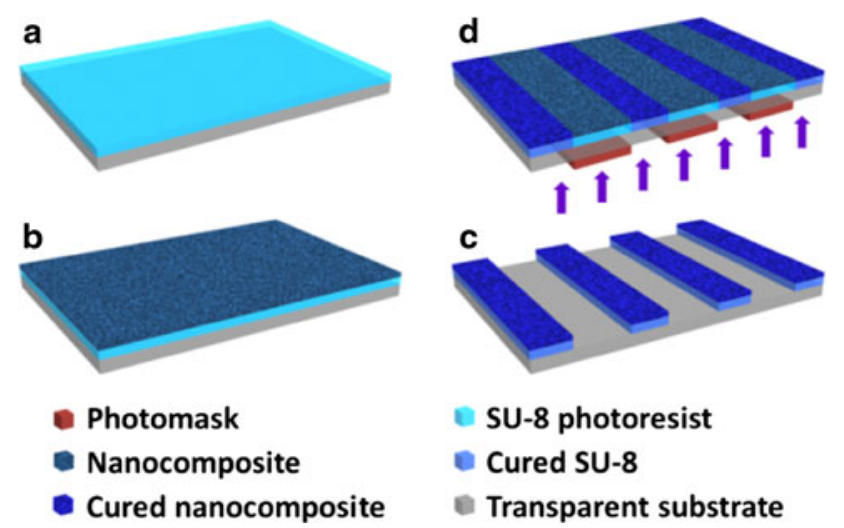

Fig. 1 Microfabrication process of the surface microfluidic networks: a spin-coating of the intermediate SU-8 layer, b spray coating of the superhydrophobic nanocomposite, $\mathbf{c}$ photopatterning of the stacked layers of SU-8 and nanocomposite, and $\mathbf{d}$ oxygen plasma treatment surface microfluidic networks in an enclosed environment, which would increase surrounding humidity, and thus reduce liquid evaporation in the open surface platform.

\subsection{Characterization of surface microflow}

Surface microflow is confined by a rigid solid-liquid interface outlined by surface wetting patterns and an elastic gasliquid interface modulated by internal fluidic pressure. Therefore, to characterize the microflow performance on the surface, the planar dimension of superhydrophobic patterns and the stereo profile of the flow will be controlled by the lithography design and external fluidic drive, respectively. In addition, a circular inlet of $1 \mathrm{~mm}$ in diameter is placed next to one end of the straight channel as the pressure indicator, while the other end is connected to an outlet reservoir with large surface area as the pressure reference approximated to the ambient pressure. Syringe pump (KD Scientific) is used to provide constant flow at various flow rates (ranging from 0.1 to $1.2 \mathrm{ml} / \mathrm{h}$ ). Under the steady-state condition, the hydraulic pressure is evaluated by measuring the interfacial curvature of the liquid profile (in the indicator reservoir) through a digital stereomicroscope placed in a horizontal position. The flow resistance is assessed by the ratio of the pressure drop to the steady-state flow rate.

\section{Results and discussion}

\subsection{Performance of superhydrophobic nanocomposite}

\subsubsection{Lithography resolution}

In the nanocomposite formula, the photosensitive SU-8 matrix is known for high optical clarity and high-definition photopatternability, whereas the embedded PTFE nanoparticles scatter the incident light and compromise the optical transmission. We have characterized the photolithographic
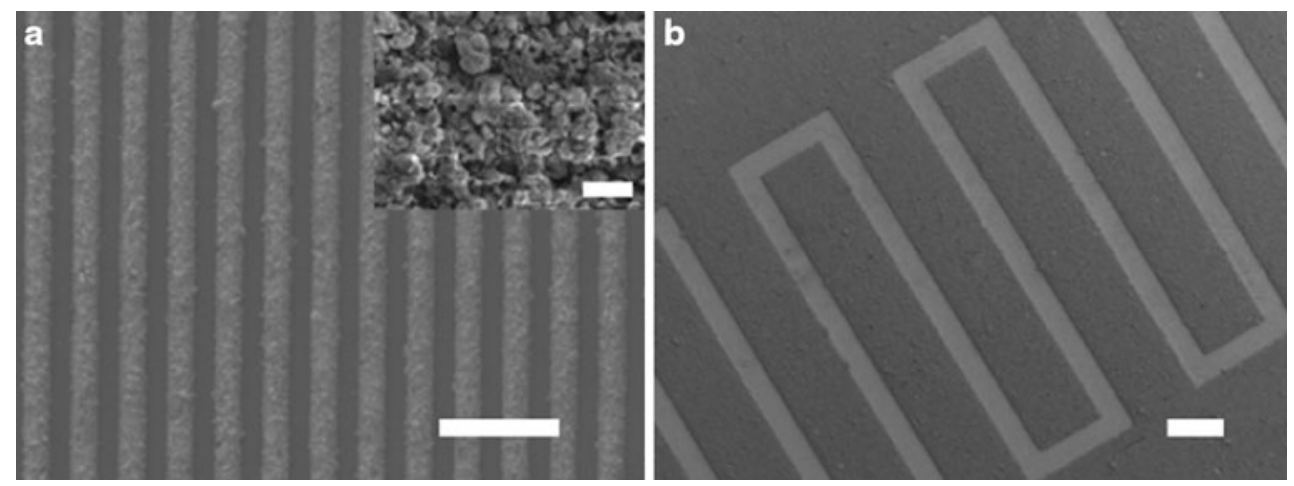

Fig. 2 SEM images of a PSN micropatterns and $\mathbf{b}$ microchannels fabricated by the single-step lithographic process along with the PSN surface morphology (in the inset) (scale bars are 10, 50, and $200 \mu \mathrm{m}$, in the inset, a, and b, respectively) 
resolution of the superhydrophobic nanocomposite formula. Figure 2 illustrates SEM images of various micropatterned features fabricated by the single-step lithography without an intermediate SU-8 layer. As can be seen, the minimal feature resolution of $10 \mu \mathrm{m}$ has been reliably achieved. Further improvements on the resolution are limited by both nanoparticle scattering and non-contact nature of the backside exposure, which can be addressed by using a thinner transparent substrate or an integrated photomask on the substrate (Choi et al. 2007).

\subsection{Nanocomposite coating}

As illustrated in Sect. 2, the thickness of superhydrophobic nanocomposite coating depends on both spray-coating process (spray volume over the surface area) and the following photo-induced curing step. Slight underexposure is performed to achieve desired nanoscopic roughness with adequate exposure of PTFE nanoparticles on the surface, which reduces the overall coating thickness. In other words, the very top part of the coating will be dissolved during the development, leading to a reduced coating thickness. Using the aforementioned protocol, (refer to Sect. 2), the coating thickness of $15 \mu \mathrm{m}$ can be reliably achieved with ultrahigh micro-nanoscale roughness as shown in the SEM image (the inset of Fig. 2a), in which PTFE nanoparticles exhibit the trend of aggregations in SU-8 matrix.

\subsection{Wettability contrast performance}

To perform surface microflow on a single planar substrate, the ultrahigh wettability contrast on the micropatterned surface becomes necessary. The superhydrophobic nanocomposite formula incorporates PTFE nanoparticles to provide both low chemical energy (free surface energy of $18.6 \mathrm{mN} / \mathrm{m}$ ) (Janczuk and Bialopiotrowicz 1990) and high

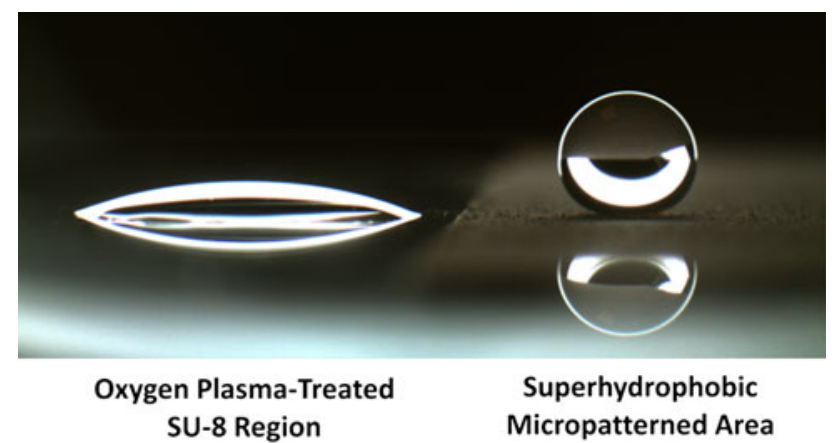

Fig. 3 Illustration of the wettability contrast between the oxygen plasma-treated SU-8 region and the superhydrophobic nanocomposite-coated area on the same glass substrate with $10 \mu \mathrm{l}$ water droplets deposited on each side nanoscopic roughness (as shown in the inset of Fig. 2a). On the other hand, glass slide is chosen as the substrate due to its intrinsic hydrophilicity (water contact angle of $10^{\circ}$ ). After the development step of the optional intermediate SU-8 layer and the top nanocomposite coating, the glass substrate is exposed as the hydrophilic floor for surface microfluidic networks. In addition, the sidewalls formed by the intermediate SU-8 layer presents hydrophilicity after oxygen plasma treatment (at $30 \mathrm{~W}$ for $30 \mathrm{~s}$ ). Figure 3 shows the wettability contrast between the oxygen plasma-treated SU-8 region and the superhydrophobic nanocompositecoated area on the same glass substrate. As can be seen, the superhydrophobicity of PTFE nanoparticles/SU-8 nanocomposite surface can be retained while the SU-8 surface renders hydrophilicity after plasma treatment. With $10 \mu \mathrm{l}$ water droplets deposited on each side, the water contact angles are measured at $18^{\circ} \pm 1^{\circ}$ and $159^{\circ} \pm 1^{\circ}$ on the hydrophilic and superhydrophobic regions, respectively.

\subsection{Surface microfluidic operations}

\subsubsection{Open surface fluidic configurations}

As illustrated in Fig. 4, surface microfluidics can be established in three different configurations, (a) the microflow confined between two surfaces of identical wetting patterns, (b) the microflow restricted between one wettability-patterned surface and one hydrophobic substrate, (c) the microflow directed on a single micropatterned substrate with ultrahigh wettability contrast. As can be seen, the first two approaches require both top and bottom surfaces for the fluidic operation (Fig. 4a, b). Moreover, the chip assembly requires precise spacing and accurate alignment, which could be time-consuming and difficult to implement. Whereas, the later design employing only a single substrate would eliminate any assembly or alignment effort, and thus,
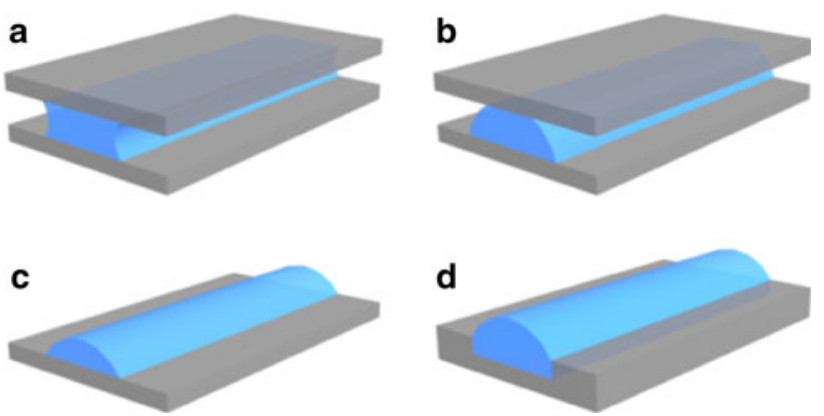

Fig. 4 Different surface microfluidic configurations: a two stacked surfaces with identical hydrophobic micropatterns, b one wettabilitypatterned surface faced to a uniform hydrophobic substrate, c a single micropatterned substrate with ultrahigh wettability contrast, and d a single wettability-patterned substrate with additive hydrophilic sidewalls 
presents apparent advantages of the open surface platform over the conventional microfluidics, as shown in Fig. 4c. However, due to high and unstable flow resistance affected largely by the exposed elastic gas-liquid interface, this approach hasn't been adequately explored for microfluidic use. In the article, the intermediate SU-8 layer is introduced to provide reliable hydrophilic sidewalls as well as enhanced interfacial adhesion, which could drastically reduce the fluidic resistance, and stabilize the surface flow according to the laminar nature of the microflow (Fig. 4d).

\subsection{Flow characteristics influenced by hydrophilic sidewall}

The influence of the hydrophilic sidewall on fluidic performance is experimentally characterized. Straight surface microchannels have been devised with the same planar dimensions (of $2 \mathrm{~cm}$ in length and $300 \mu \mathrm{m}$ in width) but various heights in the hydrophilic sidewall (of $0,15,30$, and $60 \mu \mathrm{m}$, respectively). Figure 5 shows the dependence of measured flow resistance on the pressure drop across a straight surface channel under a steady-state flow in comparison with the theoretic model, assuming an inflexible yet frictionless gas-liquid boundary, which can be then calculated as $3 \mu l / w h^{3}$ ( $\mu$ is the viscosity of the working liquid, $l, w$, and $h$ are the length, width, and height of the surface microchannel, respectively, if $w \gg h$ ) (Melin et al. 2005). The expression indicates that the height of the hydrophilic sidewall could play the most significant role (to the third power) in determining the flow resistance, therefore, the use of hydrophilic sidewalls can significantly reduce the flow resistance (i.e., doubling the height would lead to eight times reduction in the resistance if the interfacial deformation is negligible). As can be seen in Fig. 5, less resistive flow is observed in the channel with lower hydrophilic sidewalls than the predicted; in contrast, the deeper channels behave more resistive to the flow than the modeled results. The correlation between the fluidic resistance and channel height rises from combinational effects of the elastic gas-liquid interface tuned by hydraulic pressure inside as well as capillarity inside the open channels. For the same reason, the experimental results show flow resistance decreases as increment of the driven pressure, but less significant in deeper channels, which are less subject to the deformation of the flexible gas-liquid interface. In another word, the driven hydraulic pressure becomes more influential in the cross-sectional area in a shallower channel than that in a deeper channel. This can also be used to explain the considerable theoretic deviation of microflow profiles in the channel without hydrophilic sidewall (i.e., without intermediate SU-8 layer) as an extreme case $(h=0 \mu \mathrm{m})$.

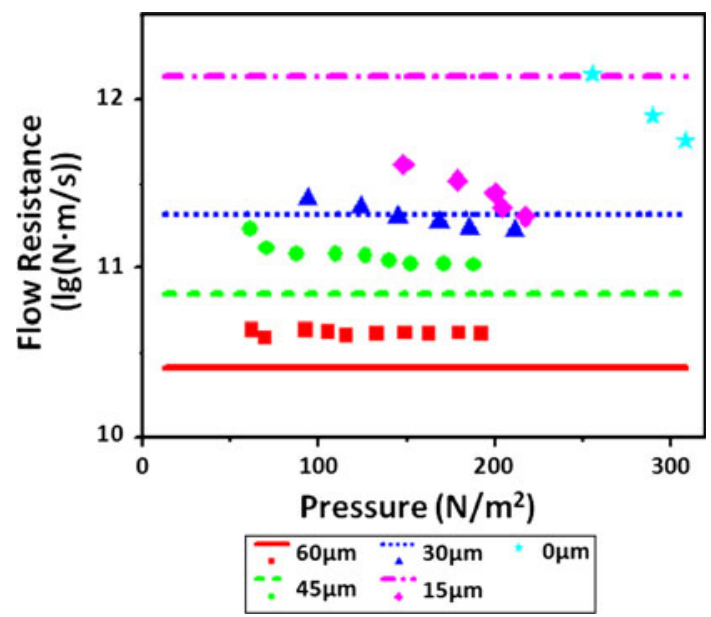

Fig. 5 Flow characteristics of surface microfluidic channels with different sidewall heights, which have identical length (of $2 \mathrm{~cm}$ ) and width (of $300 \mu \mathrm{m}$ ), in comparison with the theoretical model assuming an inflexible yet frictionless gas-liquid boundary

\subsection{Demonstration of self-propelled microfluidic networks}

Self-propelled microfluidic pump, utilizing the Ostwald's ripening phenomena of droplets (i.e., smaller liquid droplets possessing higher internal pressure automatically emerges into larger ones connected hydraulically) has been previously demonstrated in enclosed PDMS microchannels (Walker and Beebe 2002). As predicted by Laplace equation ( $\Delta p=\gamma C$, where $\gamma$ is surface tension and $C$ is curvature of the surface), the surface tension-driven microflow provides an extremely simple pumping mechanism and can be highly applicable to surface microfluidics with no external power required. In the implementation, all the fluidic components are constructed on the superhydrophobic-micropatterned surface, which further eliminate possible clogging and cavitation caused by the presence of gas bubbles. Moreover, the pressure heads established by both inlet and outlet droplets with different radii of curvature can be determined by the droplet volume and the diameter of the reservoir. In addition to the predictable pressure gradient, the straight surface microchannels can operate less dependent on the influence of the flexible gas-liquid interface under small pressure drops (e.g., Laplace pressure inside millimeter-size droplets), of which the resistance can be derived from the thin-film lubrication theory (Gennes et al. 2002). Since the Laplace pressure provides autonomous pumping mechanism, the channel resistance (e.g., channel width) can be utilized to achieve various microfluidic profiles (e.g., the peak flow rates and durations of relatively stable flow rate). Figure 6a shows three surface tension-driven pumps in parallel with connecting microchannels in different widths. Two liquid droplets with different volumes are loaded into the outlet (left: $2.3 \mu \mathrm{l}$ ) and inlet reservoirs (right: $0.45 \mu \mathrm{l}$ ) in 
sequence in each device. The inlet droplet with a smaller volume yet higher internal pressure will be spontaneously driven into the larger one at the outlet along the Laplace pressure gradient until hydraulic equilibrium is achieved. The snapshots in Fig. $6 \mathrm{~b}$ illustrate the self-propelled pumping process from the inlet droplet (in the front) to the outlet one (at the back) through a $200 \mu \mathrm{m}$-wide and $2 \mathrm{~cm}$ long microchannel (the device in front of Fig. 6a). The inlet droplet significantly shrinks and the diminished volume eventually merges into the outlet reservoir at the equilibrium under which both sides reach the same radius of curvature.

Experimental measurements have been conducted on the micropumps in comparison with the theoretic analyses, as plotted in Fig. 7. According to Laplace equation, both flow rate and driven pressure can be described as functions of the height of interfacial deformation $(H)$. Given the open surface flow resistance, the relationship between the driven pressure and flow rate is established as follows, from which $H$ can be determined:

$\frac{4 \gamma H}{H^{2}+r^{2}}-P_{\mathrm{o}}=\frac{3 \mu l}{w h^{3} 2}\left(H^{2}+r^{2}\right) \frac{\mathrm{d} H}{\mathrm{dt}}$,

where $R$ is the radius of the inlet reservoir, and $P_{\mathrm{o}}$ represents fluidic pressure in the outlet reservoir, approximated as a constant due to its relatively large volume compared with that of the inlet reservoir. Figure 7a shows the volume change of inlet droplets versus time, in which the plotted data points summarize experimental measurements in comparison with the simulated results in theory (curved lines); and furthermore, the dynamic pumping rates can be directly calculated from the volume change measurements in Fig. 7b. During the droplet merging process, the inlet one-first reduces its volume to a hemi-spherical shape (with internal pressure gradually increasing), and then diminishes to the final state (with internal pressure steadily decreasing). Measurement results in Fig. $7 \mathrm{~b}$ shows the bell-shaped trend of the dynamic flow rate versus time, in which the maximal flow rate achieves at the minimal curvature radius of the air-liquid interface of the droplet. In particular, a
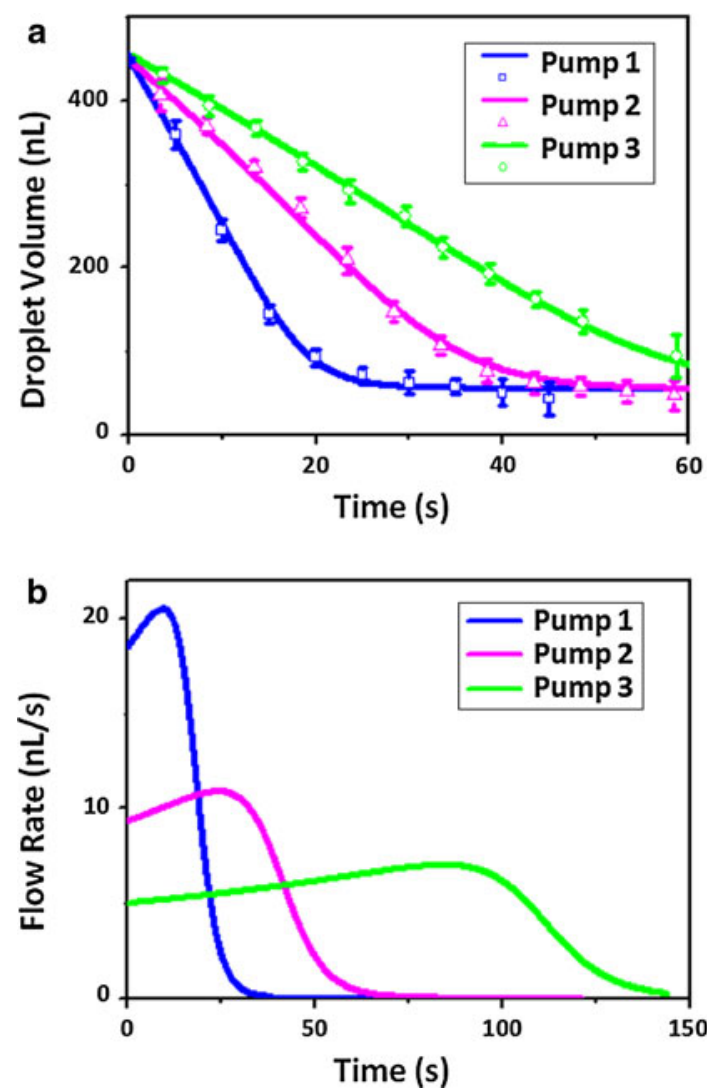

Fig. 7 Theoretical analyses and experimental measurements of the self-propelled microfluidic pumps: a the volume change of inlet droplets over time (the plotted data points summarize experimental measurements in comparison with the theoretic simulation), and $\mathbf{b}$ the dynamic pumping rates over time (the pump 1-3 indicate channel widths of 200,150 , and $100 \mu \mathrm{m}$, respectively), which are directly calculated from the volume change measurements in a

maximal flow rate of $21 \mathrm{nl} / \mathrm{s}$ has been achieved in the $200 \mu \mathrm{m}$-wide microchannel, while the $100 \mu \mathrm{m}$-wide channel offers more steady-state pumping over a 90 -s course before the inlet droplet reduces to a hemi-spherical shape. In addition, it is worth noting that the maximum pressure head, achieved when the liquid volume at the
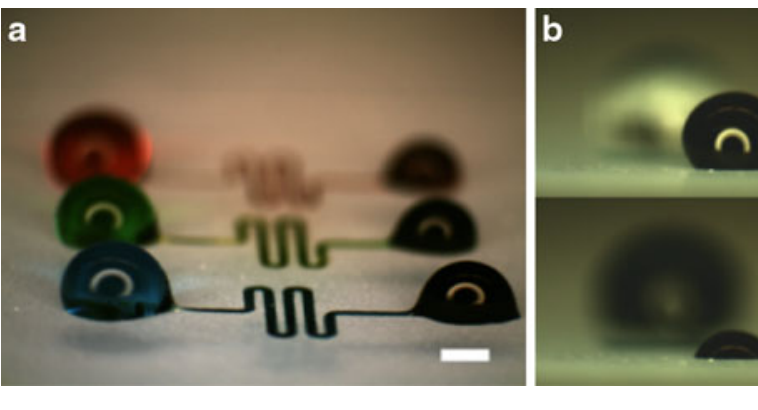

Fig. 6 a Surface tension-driven micropumps with different channel widths are loaded with colored droplets of different volumes $(0.45 /$ $2.3 \mu \mathrm{l}$ ) at each end (Note: the channel widths are 100, 150, and $200 \mu \mathrm{m}$, respectively, from the farthest to the nearest one; scale bar indicates $1 \mathrm{~mm}$ ). b The self-driven pumping process of the smaller droplet (in the front) merging into the larger one (at the back) of the pump with the connecting channel width of $200 \mu \mathrm{m}$ 
hemi-spherical shape, is determined by the diameter of reservoir according to Laplace equation, which offers unconventional geometry control on the flow characteristics (Hong and Pan 2010a, b).

\section{Conclusion}

In this article, a novel nanocomposite formula, comprising superhydrophobicity and photopatternability, has been presented to fabricate micropatterned surfaces with ultrahigh wettability contrast, which enables building surface microfluidic devices on a single-sided open platform. The one-step photolithography process delivers a feature resolution of $10 \mu \mathrm{m}$ on any planar substrate, while the superhydrophobic coating ensures extreme wettability with contact angle of above $160^{\circ}$ and long-term stability. Importantly, the micropatterned wetting boundary (i.e., interphase triple line) defines surface flow path, which serves as the foundation of surface microfluidics. Moreover, the additive intermediate SU-8 layer introduces hydrophilic sidewall with the considerably reduced flow resistance as well as improves the substrate adhesion. Surface microfluidic networks have been successfully demonstrated on the superhydrophobic patterned surfaces, where the self-propelled flow is driven by the surface tension-induced pressure gradient. Overall, the surface microfluidic systems, enabled by the single-step processing of the PSN, offers a simple yet powerful alternative to the conventional microfluidic systems, which can be widely employed in rapid-growing biomedical and chemical applications.

Acknowledgments This work is in part supported by the National Science Foundation CAREER (ECCS-0846502) and EAGER Programs (CMMI-0944353). LH acknowledges the fellowship support from China Scholarship Council (CSC). Authors would like to thank Yuzhe Ding for acquiring the SEM images.

Open Access This article is distributed under the terms of the Creative Commons Attribution Noncommercial License which permits any noncommercial use, distribution, and reproduction in any medium, provided the original author(s) and source are credited.

\section{References}

Beebe DJ, Mensing GA, Walker GM (2002) Physics and applications of microfluidics in biology. Annu Rev Biomed Eng 4:26

Bertsch A, Heimgartner S, Cousseau P, Renaud P (2001) Static micromixers based on large-scale industrial mixer geometry. Lab Chip 1:5

Besson E, Gue A-M, Sudor J, Korri-Youssoufi H, Jaffrezic N, Tardy J (2006) A novel and simplified procedure for patterning hydrophobic and hydrophilic SAMs for microfluidic devices by using UV photolithography. Langmuir 22:7

Bouaidat S, Hansen O, Bruus $\mathrm{H}$, Berendsen $\mathrm{C}$, Bau-Madsen NK, Thomsen P, Wolff A, Jonsmann J (2005) Surface-directed capillary system; theory, experiments and applications. Lab Chip $5: 10$

Choi Y, McClain MA, LaPlaca MC, Frazier AB, Allen MG (2007) Three dimensional MEMS microfluidic perfusion system for thick brain slice cultures. Biomed Microdevices 9:7

de Gennes P-G, Brochard-Wyart F, Quere D (2002) Capillarity and wetting phenomena: drops, bubbles, pearls, waves. Springer, New York

Gau H, Herminghaus S, Lenz P, Lipowsky R (1999) Liquid morphologies on structured surfaces: from microchannels to microchips. Science 283:4

Gravesen P, Branebjerg J, Jensen OS (1993) Microfluidics—a review. J Micromech Microeng 3:15

Hong L, Pan T (2010a) Photopatternable superhydrophobic nanocomposites for microfabrication. JMEMS 19:8

Hong L, Pan T (2010b) Three-dimensional surface microfluidics enabled by spatiotemporal control of elastic fluidic interface. Lab Chip 10:3271

Janczuk B, Bialopiotrowicz T (1990) The total surface free energy and the contact angle in the case of low energetic solids. J Colloid Interf Sci 140:10

Juncker D, Schmid H, Drechsler U, Wolf H, Wolf M, Michel B, de Rooij N, Delamarche E (2002) Autonomous microfluidic capillary system. Anal Chem 74:6

Lam P, Wynne KJ, Wnek GE (2002) Surface-tension-confined microfluidics. Langmuir 18:4

Lee JSH, Barbulovic-Nad I, Wu Z, Xuan X, Li D (2006) Electrokinetic flow in a free surface-guided microchannel. J Appl Phys 99:8

Melin J, van der Wijingaart W, Stemme G (2005) Behaviour and design considerations for continuous flow closed-open-closed liquid microchannels. Lab Chip 5:5

Oh CS (1999) Microfluidic electrophoresis device. US Patent 5904824

Piorek BD, Lee SJ, Santiago JG, Moskovits M, Banerjee S, Meinhart CD (2007) Free-surface microfluidic control of surface-enhanced Raman spectroscopy for the optimized detection of airborne molecules. Proc Natl Acad Sci USA 104:4

Skelley AM, Voldman J (2008) An active bubble trap and debubbler for microfluidic systems. Lab Chip 8:5

Swickrath MJ, Burns SD, Wnek GE (2009) Modulating passive micromixing in 2-d microfluidic devices via discontinuities in surface energy. Sens Actuators B 140:7

Walker GM, Beebe DJ (2002) A passive pumping method for microfluidic devices. Lab Chip 2:4

Walther F, Davydovskaya P, Zurcher S, Kaiser M, Herberg H, Gigler AM, Stark RW (2007) Stability of the hydrophilic behavior of oxygen plasma activated su-8. J Micromech Microeng 17:8

Watanabe M (2009a) Formation of a water-xylene interface in a microchannel without sidewalls. Anal Chem 81:6

Watanabe M (2009b) Surface-directed channels filled with organic solvents. Lab Chip 9:4

Watanabe M (2010) Microfluidic devices easily created using an office inkjet printer. Microfluid Nanofluid 8:6

West J, Michels A, Kittel S, Jacob P, Franzke J (2007) Microplasma writing for surface-directed millifluidics. Lab Chip 7:3

Whitesides GM (2006) The origins and the future of microfluidics. Nature 442:6

Wolfe DB, Conroy RS, Garstecki P, Mayers BT, Fischbach MA, Paul KE, Prentiss M, Whitesides GM (2004) Dynamic control of liquid-core/liquid-cladding optical waveguides. Proc Natl Acad Sci USA 101:5

Yang SY, Defranco JA, Sylvester YA, Gobert TJ, Macaya DJ, Owens RM, Malliaras GG (2009) Integration of a surface-directed microfluidic system with an organic electrochemical transistor array for multi-analyte biosensors. Lab Chip 9:5

Zhao B, Moore JS, Beebe DJ (2001) Surface-directed liquid flow inside microchannels. Science 291:4 